\title{
Prevalence of Traumatic Dental Injuries among Blind School Children in South Karnataka
}

\author{
Srinivas Danayakanapura Ramaiah ${ }^{1}$, Pradeep Kumar Maraiah ${ }^{2 *}$, \\ 1(Assistant Professor, Department Of Dentistry, Shivamogga Institute Of Medical Sciences, Shivamogga \\ RGUHS, Karnataka, India) \\ 2(ASSOCIATE PROFESSOR, DEPARTMENT OF OPHTHALMOLOGY, SHIVAMOGGA INSTITUTE OF MEDICAL SCIENCES, \\ SHIVAMOGGA, RGUHS, Karnataka, India)
}

\begin{abstract}
:
Background and Objectives: Traumatic Dental Injuries (TDI) is emerging as an important dental cause of public health concern. Visually impaired children are more prone for these injuries. Proper approach to the management requires knowledge about the extent, distribution and associated risk factors of this specific condition. So, the study was done to assess the prevalence of TDI among students of blind school in Shivamogga town, Karnataka, India.

Materials and methods: A cross sectional study was conducted in a total of 80 students of 5 to 16 years age group. All students were divided into partially blind and totally blind groups based on WHO criteria for definition of blindness. Data were collected through WHO oral health survey form and clinical examination regarding gender, age, number of teeth injured, lip coverage, maxillary overjet and pattern of teeth injury. Collected data were analyzed using Microsoft Excel and SPSS software.

Result: The prevalence of dental trauma in the present study was $37.5 \%$. Anterior teeth only were injured in this study. Children with maxillary overjet \& inadequate lip coverage had more dental injuries. Enamel fracture was the most common type of fracture recorded. None of the children had undergone any dental treatment for their injuries.
\end{abstract}

Conclusion: Common occurrence of dental trauma \& lack of treatment among blind school children necessitates creating awareness, health education and periodic screening for appropriate management.

Keywords: traumatic dental injury, visually impaired, maxillary overjet, lip coverage, teeth fracture, blind school children.

\section{Introduction}

Traumatic dental injuries (TDI) are common day to day occurrence in children \& adolescents [1]. These injuries can cause irreparable dental damage not only at the time of event but also during post treatment period [2]. Despite high prevalence of dental trauma, less attention has been given to TDI, its etiology \& prevention [3]. In recent years there is a decline in prevalence \& severity of dental caries seen in many countries\& TDI is emerging as a serious oral health problem in children $[2,4]$.

More than $20 \%$ of children experience damage to their permanent dentition by the age of 14 years, the peak incidence at the age of 8-10 years [3]. These TDI not only cause problems with eating food \& speech but also have a serious deleterious effect on aesthetics, behavior \& overall personal development of the child [1, 4].

Review of earlier literature shows that various causes for TDI IN normal children include fall, play related, collision, road traffic accidents, sports, violence \& also child abuse [1,3]. Increased maxillary overjet with protrusion \& inadequate lip coverage are implicated as important intrinsic risk factors for TDI. It has also been observed that anterior teeth are more commonly involved in TDI [1,2].

Data from earlier studies suggests that TDI prevalence in children with various disabilities is higher than that of normal children $[5,6,7$, and 8]. However there are limited number of literature on prevalence of TDI in visually impaired children \& adolescents in India \& also worldwide. Hence the present study was undertaken to assess the prevalence of TDI among blind school children in Shivamogga town, Karnataka, India.

\section{Materials \& Methods}

A cross sectional study was conducted in a private blind school in Shimoga town, Karnataka, India. The study sample consisted of 80 students of the blind school aged between 5 to 16 years. Prior written consent was obtained from the Principal/Administrator of the school after explaining the aims \& objectives of the study. Visual acuity was recorded using Snellen's illiterate E chart with appropriate spectacle correction.

All the children were divided into totally blind \& partially blind groups according to WHO definition of blindness [9]. Based on the best corrected visual acuity children were grouped into following categories: 
Totally blind: visual acuity worse than counting fingers at three meters (3/60) including presence or absence of light perception in the better eye.

Partially blind: visual acuity worse than $6 / 18$ but better than $3 / 60$ in the better eye.

A printed proforma was designed according to WHO Oral Health Assessment Form for Children, 1993[10]. A single examiner examined all the children in broad day light after seating them on a chair. Before commencement of intraoral examination lip coverage of upper incisors was observed in resting position without the awareness of the child. Lip coverage was considered inadequate if the lips failed to cover upper incisors with major part of crown exposed. Also maxillary overjet was noted in each child \& an overjet equal to or more than $3 \mathrm{~mm}$ was considered significant. Later a detailed intraoral examination was done to note any evidence of dental trauma \& tooth fracture among all the teeth. Tooth fractures were recorded as per WHO Oral Health Survey Guidelines.

\section{Results}

A total of 80 children were examined their age limit ranging from 5 years to 16 years, mean age was 13.98 years with standard deviation of 3.639. More than half of children (51.3\%) were in 9 to 12 age group. Among the total 80 children 50(62.5\%) were males \& 30(37.5\%) were females.

In the present study nine children $(11.3 \%)$ were partially blind and 71 were totally blind $(88.8 \%)$ according to WHO definition. Assessment of fracture occurrence according to severity of blindness shows $22.2 \%$ of partially blind children and $39.4 \%$ of totally blind children had teeth fracture. However association of severity of blindness with fracture occurrence was not statistically significant $(\mathrm{p}=0.315)$.

Teeth fracture were noted in 30(37.5\%) out of 80 children. All 30 children had anterior teeth fracture out of which single tooth fracture was found in $23(76.67 \%) \&$ remaining seven had multiple teeth fracture involving central incisors. Right Central Incisor (53.4\%) was most commonly involved among all teeth fractures followed by left central incisors (23.3\%), central incisors (13.3\%), left central with lateral incisors $(6.7 \%) \&$ right central with lateral incisors (3.3\%).

Table1: Type of teeth involved in fracture

\begin{tabular}{|c|c|c|c|}
\hline \multicolumn{2}{|c|}{ Teeth Involved } & Frequency & Percent \\
\hline \multirow{4}{*}{} & BC & 4 & 13.3 \\
\cline { 2 - 4 } & RC & 16 & 53.4 \\
\cline { 2 - 4 } & LC & 7 & 23.3 \\
\cline { 2 - 4 } & LC,LL & 2 & 6.7 \\
\cline { 2 - 4 } & RC,RL & 1 & 3.3 \\
\hline
\end{tabular}

(Legends: $\mathrm{BC}=$ both central incisors, $\mathrm{RC}=$ right central incisor, $\mathrm{LC}=$ left central incisor, $\mathrm{LL}=$ left lateral incisor, $\mathrm{RL}=$ right lateral incisor)

Normal Maxillary Overjet was noted in 72 of 80 children (90\%) while abnormal Overjet of more than or equal to $3 \mathrm{~mm}$ was found in remaining eight $(10 \%)$. Seven out of eight children with abnormal overjet $(87.5 \%)$ had teeth fracture \& only 23 of $72(31.9 \%)$ with normal overjet had fracture teeth. This association of Maxillary overjet with teeth fracture is statistically significant with $p=0.002$ ( $p$ value $<0.005$ is set as statistically significant).

Table2. Association of teeth fracture with maxillary overjet.

\begin{tabular}{|c|c|c|c|c|c|}
\hline \multicolumn{6}{|c|}{ Association of teeth fracture with overjet } \\
\hline & & & \multicolumn{2}{|c|}{ Fracture } & \multirow{2}{*}{ Total } \\
\hline & & & Present & Absent & \\
\hline \multirow{4}{*}{ Overjet } & \multirow{2}{*}{ Normal } & Frequency & 23 & 49 & 72 \\
\hline & & $\%$ & $31.9 \%$ & $68.1 \%$ & $100.0 \%$ \\
\hline & \multirow{2}{*}{ Abnormal } & Frequency & 7 & 1 & 8 \\
\hline & & $\%$ & $87.5 \%$ & $12.5 \%$ & $100.0 \%$ \\
\hline \multirow{2}{*}{\multicolumn{2}{|c|}{ Total }} & Frequency & 30 & 50 & 80 \\
\hline & & $\%$ & $37.5 \%$ & $62.5 \%$ & $100.0 \%$ \\
\hline \multicolumn{2}{|c|}{ Chi square $=9.481$} & $\mathrm{Df}=1$ & \multicolumn{2}{|c|}{$\mathrm{p}$-value $=0.002$} & Significant \\
\hline
\end{tabular}

Lip coverage was observed in all children without their knowledge. Among the total of 80, seven children $(23.3 \%)$ had inadequate lip coverage as evidenced by failure of lips to cover upper incisors $\&$ major part of crown exposed in resting position. All these children with inadequate lip coverage had teeth fracture $(100 \%)$ while out of 73 with adequate lip coverage only $23(31.5 \%)$ had teeth fracture. This association of inadequate lip coverage with fracture was statistically highly significant $(\mathrm{p}<0.001)$. 
Table3: Association of fracture with lip coverage

\begin{tabular}{|c|c|c|c|c|c|}
\hline \multicolumn{6}{|c|}{ Association of Fracture with lip coverage } \\
\hline & & & fracture & & Total \\
\hline & & & Present & Absent & \\
\hline \multirow{4}{*}{ Lip coverage } & \multirow{2}{*}{ Adequate } & Frequency & 23 & 50 & 73 \\
\hline & & $\%$ & $31.5 \%$ & $68.5 \%$ & $100.0 \%$ \\
\hline & \multirow{2}{*}{ Inadequate } & Frequency & 7 & 0 & 7 \\
\hline & & $\%$ & $100.0 \%$ & $0.0 \%$ & $100.0 \%$ \\
\hline \multirow{2}{*}{\multicolumn{2}{|c|}{ Total }} & Frequency & 30 & 50 & 80 \\
\hline & & $\%$ & $37.5 \%$ & $62.5 \%$ & $100.0 \%$ \\
\hline \multicolumn{2}{|l|}{$p$-value $=0.001$} & Significant & & & \\
\hline
\end{tabular}

Pattern of teeth involvement reveals that Enamel involvement is most common type of lesion $(60 \%)$ among all fractures. Combined Enamel \& Dentine fracture is second common type (33.3\%) followed by pulp involvement \& missing tooth due to trauma seen in one case each. There were no cases of treated injury in the study group. Among 23 single tooth fractures again Enamel fracture type was most common (78.3\%) while all seven multiple teeth fractures had combined Enamel \& Dentine fracture.

Table4: Fracture grading according to WHO classification

\begin{tabular}{|l|l|l|l|}
\hline Fracture grading & 0 & Frequency & Percent \\
\hline \multirow{5}{*}{ WHO classification } & 2 & 50 & 62.5 \\
\cline { 2 - 5 } & 3 & 18 & 22.5 \\
\cline { 2 - 5 } & 4 & 10 & 12.5 \\
\cline { 2 - 5 } & 5 & 1 & 1.3 \\
\cline { 2 - 4 } & Total & 1 & 1.3 \\
\hline
\end{tabular}

Table5: Comparison of type of teeth fracture according to WHO classification

\begin{tabular}{|c|c|c|c|c|c|c|}
\hline & & \multicolumn{4}{|c|}{ Type of Teeth Fracture } & \multirow[t]{2}{*}{ Total } \\
\hline & & Type 2 & Type 3 & Type 4 & Type 5 & \\
\hline \multirow{2}{*}{ Single tooth } & Frequency & 18 & 3 & 1 & 1 & 23 \\
\hline & $\%$ & $78.3 \%$ & $13.0 \%$ & $4.3 \%$ & $4.3 \%$ & $100.0 \%$ \\
\hline \multirow{2}{*}{ Multiple teeth } & Frequency & 0 & 7 & 0 & 0 & 7 \\
\hline & $\%$ & $0.0 \%$ & $100.0 \%$ & $0.0 \%$ & $0.0 \%$ & $100.0 \%$ \\
\hline \multirow{2}{*}{ Total } & Frequency & 18 & 10 & 1 & 1 & 30 \\
\hline & $\%$ & $60.0 \%$ & $33.3 \%$ & $3.3 \%$ & $3.3 \%$ & $100.0 \%$ \\
\hline
\end{tabular}

\section{Discussion}

Prevalence of TDI in normal children differs considerably among various epidemiological studies across the country $\&$ around the world. Prevalence of TDI within the country ranges from 8.79 to $18.25 \%$ [1, 2 , $3,4]$. This wide variation among the studies can be explained by differences in various factors like trauma classification used, dentition status of children, geographical variation \& also behavioral differences [1, 3, 5].

Studies assessing prevalence of TDI among visually impaired children also have found wide variation ranging from $9 \%$ to $32.5 \%[5,6,8,9]$. However prevalence of TDI in our study is consistent with Bhat et al (32.5\%) [6] \& Agarwal et al (34.95\%) [8]. Children with disabilities are more prone for TDI compared to their normal counterpart $[5,8]$. Visually impaired individuals suffer from many oral health problems including orofacial trauma, dental caries \& periodontal disease. Among these orofacial trauma constitutes the main health hazard for the visually impaired. Also these individuals receive less oral health care compared to subjects from general population [8].

In the present study majority of children $(88.8 \%)$ were totally blind while a study in blind school from Rajasthan showed only $26.2 \%$ were totally blind in their study [8]. The same study also found occurrence of teeth fracture in $58.33 \%$ of partially blind but the present study showed only $22.2 \%$ of partially blind children sustaining fracture. However presence of teeth fracture in totally blind children of present study (39.4\%) was similar to above study (41.67\%) and study by O'Donnell (36.4\%) [11].This difference can be explained partly by variations in admission criteria among different blind schools with respect to age of admission and severity of blindness. 
Male gender is predisposed for TDI in many earlier studies both in normal \& visually impaired children. However AK Murthy et al [5] have found no gender predisposition for TDI among visually impaired $\&$ interestingly female predominance was found by Agarwal et al [8]. This male predisposition pattern can be explained by the fact that boys engage more in leisurely physical activities, aggressive sports \& also greater risk taking behavior than girls. Also girls are said to be more mature in their behavior $[1,2,3,4,6]$.

Many earlier studies have recognized the importance of intrinsic risk factors like maxillary overjet $\&$ lip coverage on the occurrence of TDI [12]. There is a significant association between TDI \& maxillary overjet $>3 \mathrm{~mm}[1,2,3,4]$. Also studies have concluded that frequency of TDI increases proportionately in relation to increased overjet. This positive correlation is also seen in visually impaired children $[6,8]$. In the present study $87.5 \%$ of visually impaired students with abnormal overjet had teeth fracture which correlates with earlier studies by Bhat et al (70.4\%) [6] \& Agarwal et al (85.7\%) [8].

Inadequate lip coverage is another important intrinsic risk factor for TDI both in normal \& visually impaired children $[1,3,4,8]$. In the present study all seven children with inadequate lip coverage had TDI. This value is higher than Agarwal et al who found that up to $78.6 \%$ of visually impaired children had TDI [8]. But in contrast to above reports Bhat et al have reported that fracture teeth was independent of lip coverage [6]. It has been suggested that protective effect of lip closure along with adequate contact of maxillary \& mandibular teeth in normal occlusion decreases the impact force of trauma $[1,3,4]$.

Only Anterior teeth were involved in the present study which is consistent with reports of earlier studies showing that central incisors are more commonly involved in TDI $[1,2,3,4,5,7,8]$. The reason for this predisposition is their vulnerable position in the oral cavity \& frequent protrusion of incisors which are more commonly involved in TDI [8].

Pattern of teeth involvement in the present study shows that enamel fracture is the most common injury $(60 \%)$ although a study in visually impaired children showed combined dentine \& enamel fracture as the common type of injury [8]. However many earlier studies in normal children have also shown enamel fracture as the most common injury pattern $[1,2,3,4,5] \&$ further studies in visually impaired are required to ascertain this fact.

None of the students in the present study received any form of treatment for their teeth fractures. This indicates poor awareness among parents \& caretakers in blind schools regarding TDI. Even normal children are also deprived of management of teeth fracture as evidenced by studies showing less than $5 \%$ of these children receiving any dental treatment. These studies have implicated not only lack of awareness but also economic \& social factors [2,3]. Also first attendance by a dental surgeon is delayed [13].

\section{Conclusion}

Traumatic Dental Injuries are the most neglected oral health aspect in children. Children with visual impairment are more vulnerable for TDI. In an effort to reduce the prevalence of TDI the present study recommends the following strategies:

1. Health education for visually impaired children, parents \& care takers of blind schools including teachers regarding etiology of TDI, its prevention \& availability of treatment.

2. Eliminating or reducing the severity of intrinsic oral risk factors like maxillary overjet by appropriate orthodontic treatment.

3. Conducting periodic screening programs in blind schools for early recognition of TDI \& also to identify children with high anatomic \& behavioral risk for TDI for appropriate preventive measures.

\section{References}

[1]. Kalaskar RR, Kalaskar AR, Wankhade RM, Mehta JD. Evaluation of prevalence, etiological \& risk factors of traumatic dental injury in 12-14 years old school going children of Central India. SRM J of research in dental sciences. 2014:5; 2; 73-77

[2]. Govindarajan M, Reddy VN, Ramalingam K, Durai KS, Rao PA, Prabhu A. Prevalence of traumatic dental injuries to the anterior teeth among three to thirteen-year-old school children of Tamilnadu. Contemporary clinical dentistry. 2012:3;2;164-167

[3]. Ahlawat B, Kaur A, Thakur G, Mohindroo A. Anterior tooth trauma: A most neglected oral health aspect in adolescents. IJOR. 2013:4; 1; 31-37

[4]. Patel MC, Sujan SG. The prevalence of traumatic dental injuries to permanent anterior teeth and its relation with predisposing risk factors among 8-13 years school children of Vadodara city: An epidemiological study. Journal of Indian society of pedodontics \& preventive dentistry. 2012:30;2;151-157

[5]. Murthy AK. Chandrakala B, Pramila M, Ranganath S. Dental trauma in children with disabilities in India: A comparative study. European Archives of Pediatrics Dentistry.2013:14; 4; 221-225

[6]. Bhat N, Agarwal A, Nagarajappa R, Roy SS, Singh K, Chaudhary H, Asawa K et al. Teeth fracture among visually impaired \& sighted children of 12 to 15 years of age groups of Udaipur city, India-a comparative study. 2011:27;5;389-392

[7]. AlSarheed M, Bedi R, Hunt NP. Traumatized permanent teeth in 11-16-year-old Saudi Arabian children with a sensory impairment attending special schools. Dent Traumatolgy.2003:19; 3; 123-125

[8]. Agarwal A, Bhatt N, Chaudhary H, Singh K, Mishra P, Asawa K. Prevalence of anterior teeth fracture among visually impaired individuals, India. IJDR.2013:24; 6; 664-668

[9]. apps.who.int/classifications/icd10/chapter 7/H53-H54 
[10]. World Health Organization. Implementing the survey. Oral Health Surveys: Basic Methods. $4^{\text {th }}$ ed. Geneva: World Health Organization. 1999. 16-20

[11]. O'Donnell d. The prevalence of nonrepaired fractured incisors in visually impaired Chinese children and young adults in Hong Kong. Quintessence Int 1992; 23:363-65

[12]. Glendor U. Aetiology \& risk factors related to traumatic dental injuries-a review of literature. Dental Traumatology. 2009:25; 1 ; 19-31

[13]. 13.Ferreira MCG, Guare RO, Prokopowitsch I, Santos MTBR. Prevalence of dental trauma in individuals with special needs. Dental Traumatology. 2011:27; 2; 113-116 